\title{
Manejo del traumatismo de aorta torácica en cinco pacientes politraumatizados
}

\author{
P. ORTIZ ${ }^{a}$, A. TACHÉa , J.M. SIRVENT ${ }^{a}$, A. BONET'a M. FEBRER ${ }^{b}$ Y O. ANDRÉS ${ }^{c}$ \\ aServicio de Medicina Intensiva. ' Servicio de Radiodiagnóstico. `Servicio de Cirugía Vascular. \\ Hospital Universitari de Girona Doctor Josep Trueta. Girona. España.
}

El traumatismo de aorta torácica es una lesión asociada a una alta morbimortalidad en el lugar del accidente. En los años noventa se generalizó el tratamiento mediante la colocación de un stent endovascular con buenos resultados. En este trabajo presentamos una serie de cinco casos clínicos de pacientes ingresados en la Unidad de Cuidados Intensivos de nuestro hospital durante el año 2006. De un total de 619 pacientes ingresados en el mismo periodo, 121 (19,5\%) fueron traumáticos y $5(4,13 \%)$ sufrían traumatismos de aorta torácica. Todos los pacientes fueron diagnosticados por tomografía computarizada helicoidal torácica al ingreso. Cuatro pacientes tenían un pseudoaneurisma de aorta y fueron intervenidos. Tres recibieron tratamiento endovascular dentro de las $\mathbf{3 6}$ primeras horas y el otro al vigesimoctavo día del ingreso. El quinto paciente presentó una lesión de la íntima y no se intervino. La evolución posterior fue buena en todos los casos.

PALABRAS CLAVE: traumatismo de aorta torácica, tratamiento endovascular, politraumatismo.

\section{MANAGEMENT OF THORACIC AORTA TRAUMATISM IN 5 MULTIPLE TRAUMATIZED PATIENTS}

Thoracic aorta traumatism is a lesion associated to high morbidity-mortality at the site of the accident. In the 90 's, treatment by placement of an

Correspondencia: Dra. P. Ortiz Ballujera.

Servicio de Medicina Intensiva (UCI)

Hospital Universitari de Girona Doctor Josep Trueta.

Avda. de França, s/n

17007 Girona. España.

Correo electrónico: ballujera@ hotmail.com

Manuscrito aceptado el 10-VII-2007. endovascular stent was generalized with good results. In this work, we present a series of 5 clinical cases of patients admitted to the Intensive Care Unit of our hospital during the year 2006. Out of a total of 619 patients admitted in the same period, $121(19.5 \%)$ were traumatic and $5(4.13 \%)$ had thoracic aorta traumatisms. All of the patients were diagnosed by thoracic helical computed tomography on admission. Four patients had a pseudoaneurism of the aorta and underwent an operation. Three received endovascular treatment within the first $\mathbf{3 6}$ hours and the fourth on day $\mathbf{2 8}$ of admission. The fifth patient had a lesion of the intima and was not operated on. Posterior evolution was good in all the cases.

KEY WORDS: thoracic aorta traumatism, endovascular treatment, multiple traumatism.

\section{INTRODUCCIÓN}

El traumatismo de aorta torácica es una lesión infrecuente en la clínica debido a su alta mortalidad (80-90\%) en el lugar del accidente. Su frecuente asociación a traumatismos en otras localizaciones le añade una mayor morbimortalidad ${ }^{1}$. En pacientes con sospecha de esta lesión se ha de realizar una tomografía computarizada helicoidal torácica (TACHT) o un ecocardiograma transesofágico (ECOT) para descartarla $^{2,3}$. El manejo médico se centra en el control estricto de la tensión arterial dando prioridad al tratamiento de las lesiones asociadas que comprometan la vida. La colocación de un stent de forma percutánea permite los mismos resultados que con la cirugía abierta, pero con menos complicaciones asociadas, y mejores resultados que cuando el manejo es exclusi- 
TABLA 1. Resumen de datos de los 5 pacientes

\begin{tabular}{|c|c|c|c|c|}
\hline Caso & Edad & ISS* & Tiempo diagnóstico/intervención (horas) & Lesiones asociadas \\
\hline 1 & 27 & 42 & 672 & $\begin{array}{l}\text { Rotura esplénica. Lefort III. Laceración pulmonar bilateral. Contusión } \\
\text { hepática }\end{array}$ \\
\hline 2 & 31 & 16 & 8 & Hematoma mediastínico. Hemotórax bilateral \\
\hline 3 & 40 & 38 & 10 & $\begin{array}{l}\text { TCE leve. Hemotórax bilateral. Laceración hepática. Contusión suprarrenal. } \\
\text { Fractura de acetábulo }\end{array}$ \\
\hline 4 & 35 & 43 & - & $\begin{array}{l}\text { TCE grave. Fractura de clavícula y escápula izquierda, } 1 .^{\text {a }} \text { costilla bilateral, } \\
2 .^{a} \text { y } 3 .^{a} \text { costilla izquierda. Fractura de pelvis }\end{array}$ \\
\hline 5 & 45 & 32 & 36 & $\begin{array}{l}\text { Contusión pulmonar bilateral. Contusión hepatoesplénica. Hematoma } \\
\text { suprarrenal izquierdo }\end{array}$ \\
\hline
\end{tabular}

*Injury Severity Score; TCE: traumatismo craneoencefálico.

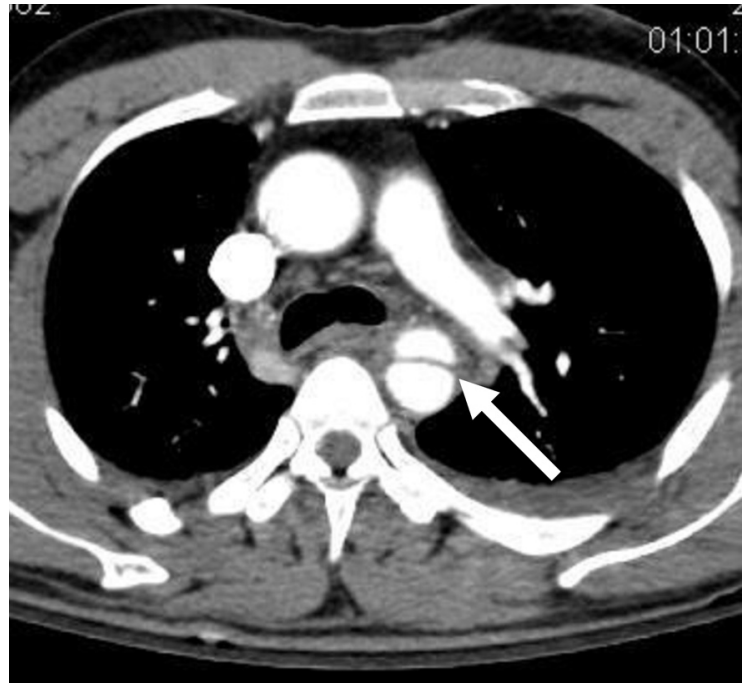

Figura 1. Corte axial que muestra colgajo intimal (flecha blanca).

vamente médico ${ }^{4}$. Según los estudios realizados, es controvertido el momento idóneo de la intervención ${ }^{5}$. La gravedad de las lesiones asociadas no ha permitido en algunos casos un tratamiento quirúrgico con el que se obtengan buenos resultados ${ }^{6}$. Hay lesiones de aorta menos graves que se pueden tratar de forma conservadora $^{7,8}$.

\section{CASO 1}

Se trata de un varón de 27 años de edad que ingresa por politraumatismo después de precipitarse desde 15 metros. Es atendido en el lugar del accidente con Glasgow Coma Scale de 6 puntos y se consigue estabilidad hemodinámica. En la TACHT se diagnosticó un seudoaneurisma de aorta torácica en el istmo y hematoma periaórtico acompañante. Las lesiones asociadas de todos los casos se describen en la tabla 1.

El paciente se trató con labetalol para el control estricto de la tensión arterial. Los ECOT a las 24 horas y a la semana de seguimiento de la lesión aórtica no presentaron cambios. Al noveno día fue dado de alta de la Unidad de Cuidados Intensivos (UCI) y al vigesimoctavo día del accidente se le colocó una en- doprótesis en la aorta torácica descendente por la vía ilíaca primitiva izquierda con oclusión asintomática de la arteria subclavia izquierda.

\section{CASO 2}

El paciente era un varón de 31 años de edad que sufrió un accidente de motocicleta. La radiografía de tórax mostraba un ensanchamiento mediastínico. La TACHT al ingreso mostró un seudoaneurisma de aorta torácica distal a la salida de la arteria subclavia izquierda con un importante hematoma periaórtico y mediastínico asociado con hemotórax bilateral (fig. 1).

Ingresó en la UCI, donde se consiguió un control estricto de la tensión arterial. A las 8 horas del traumatismo se colocó una endoprótesis en la aorta torácica descendente por vía ilíaca izquierda. La arteria subclavia izquierda quedó parcialmente ocluida sin repercusión clínica. A las 24 horas fue dado de alta a planta y a la semana se le dio el alta hospitalaria, ya que estaba asintomático.

\section{CASO 3}

Se trata de un varón de 40 años que sufrió un accidente de coche. La TACHT al ingreso mostraba un seudoaneurisma de la aorta torácica a la salida de la arteria subclavia izquierda con un hematoma periaórtico extendido al mediastino posterior. El paciente ingresó en la UCI en respiración espontánea con tensión arterial sistólica (TAS) inferior a $120 \mathrm{mmHg}$. Se llevó a cabo un tratamiento conservador de las lesiones asociadas.

A las 10 horas del ingreso en la UCI se le colocó una endoprótesis en la aorta torácica descendente por vía femoral izquierda, con oclusión parcial de la arteria subclavia izquierda (fig. 2). Fue dado de alta a planta a las 48 horas. Siguió controles con TACHT a los 7 días, al mes y a los 45 días.

A los dos meses del accidente presentó una pericarditis aguda y ausencia de pulso radial izquierdo sin repercusión clínica. La TACHT mostraba una correcta localización de la endoprótesis.

\section{CASO 4}

El paciente era un varón de 35 años de edad que sufrió un accidente de motocicleta. La TACHT al in- 


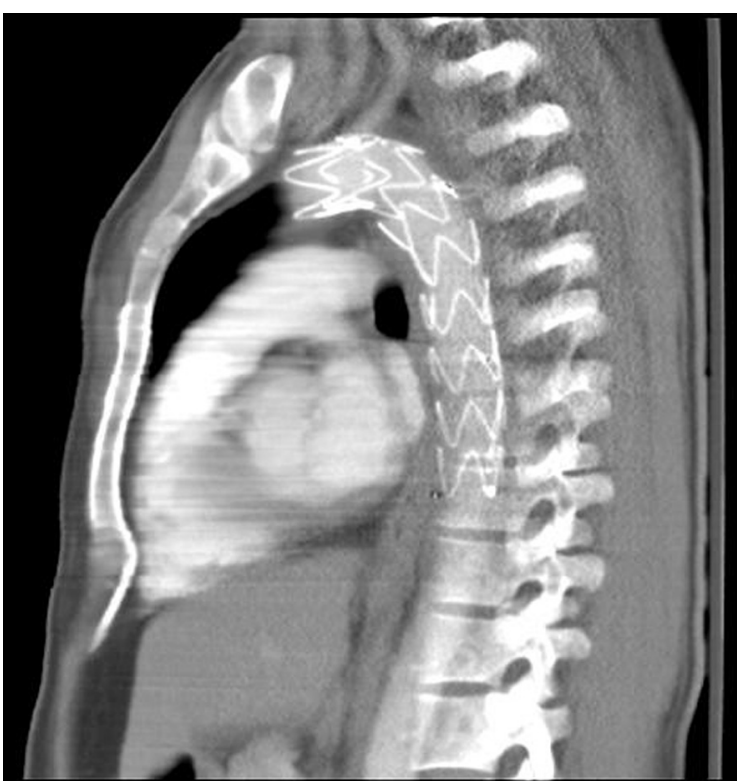

Figura 2. Corte sagital que muestra correcta colocación de prótesis endovascular.

greso mostraba una laceración intimal de $7 \mathrm{~mm}$ en la aorta torácica después de la salida del tronco braquiocefálico.

Al paciente se le realizó una craneotomía parietal derecha descompresiva y la evacuación de un hematoma subdural de forma urgente. Se inició tratamiento con labetalol endovenoso para el control estricto de la TAS y se priorizó el manejo de las lesiones asociadas debido a su gravedad. El ECOT a la semana mostró mejoría respecto al del ingreso, a los 15 días la angioresonancia magnética (angio-RMN) informó de una normal distribución de la salida de los troncos supraaórticos sin imágenes sugerentes de rotura traumática de la aorta. Al mes del ingreso fue dado de alta domiciliaria.

\section{CASO 5}

Se trata de un varón de 45 años que sufrió un accidente de coche. En la valoración inicial estaba estable hemodinámicamente. La TACHT corporal al ingreso mostraba: seudoaneurisma de la aorta torácica distal a la arteria subclavia izquierda con sangrado periaórtico. Ingresó en la UCI, donde se inició el tratamiento médico. A las 36 horas se le realizó una reparación quirúrgica con endoprótesis en la aorta torácica descendente por la vía femoral izquierda. Hubo una oclusión asintomática de la arteria subclavia izquierda.

La TACHT de control a los 6 días mostraba una correcta localización de la endoprótesis. Al décimo día presentó una pericarditis aguda y fue dado de alta al decimosexto día, asintomático.

\section{DISCUSIÓN}

A pesar de que el traumatismo de aorta es una lesión poco frecuente encontramos una alta incidencia en la serie presentada. El ser el único hospital de referencia para traumáticos de la provincia de Gerona, siendo además ésta una región con muchos accidentes de tráfico, podría ser una explicación. Además, a todos los traumatismos graves se les aplica un protocolo de una TACHT, una tomografía computarizada helicoidal abdominal y otra cerebral al ingreso si las condiciones del paciente lo permiten.

Los traumatismos de aorta frecuentemente se asocian a lesiones en otras localizaciones: contusión-laceración pulmonar, hemo-neumotórax, fracturas costales múltiples, fracturas de esternón, fractura de pelvis, lesiones de órganos sólidos intraabdominales, traumatismo craneoencefálico (TCE) y otras fracturas óseas. Las lesiones asociadas son las que determinan la gravedad del paciente y las decisiones terapéuticas $^{6}$.

Todos nuestros pacientes presentaron lesiones torácicas, tres de ellos lesiones de órgano sólido intrabdominal y dos pacientes TCE y lesiones óseas asociadas.

La TACHT con contraste es la prueba diagnóstica definitiva en el paciente hemodinámicamente estable y además permite una valoración de las lesiones asociadas. La presencia de signos directos de rotura aórtica (colgajo intimal, contorno aórtico irregular, trombo luminal y extravasación de contraste aórtico) son altamente específicos, por lo que no es necesaria la realización de otras pruebas para el manejo de estos pacientes ${ }^{3,9}$. Se considera que esta prueba tiene un valor predictivo negativo del $100 \%{ }^{10}$.

El ECOT es la prueba de elección inicial en los pacientes hemodinámicamente inestables, dada su facilidad de realización a la cabecera del enfermo. Ambas pruebas podrían ser igualmente válidas para el diagnóstico inicial y la elección de una u otra estaría determinada por las lesiones asociadas del paciente y su estabilidad hemodinámica ${ }^{2,3}$.

La alta mortalidad asociada a esta lesión obliga, por tanto, a un diagnóstico precoz para poder orientar el tratamiento adecuado.

En estos pacientes se han de priorizar las lesiones asociadas que comprometan la vida. El objetivo es el estricto control de la tensión arterial entre 120 y $100 \mathrm{mmHg}$ o inferior, siempre que se asegure la perfusión de los órganos vitales ${ }^{6,11}$. Los fármacos de elección serían los bloqueantes beta y, en caso de estar contraindicados, los antagonistas de los canales del calcio.

Hirose et al publicaron el tratamiento no quirúrgico de esta lesión en 7 pacientes con un $29 \%$ de mortalidad debida a las lesiones asociadas ${ }^{6}$. En una serie de 44 pacientes con traumatismo de aorta 5 recibieron tratamiento médico con una supervivencia del $100 \%{ }^{12}$.

En 1994 Dake et al ${ }^{13}$ publicaron el éxito del tratamiento endovascular para la reparación de aneurismas de aorta torácica.

Todos los trabajos revisados concluyen que este tratamiento es tan eficaz como la cirugía abierta ${ }^{4,5,14,15}$. Esta técnica permite evitar las complicaciones asociadas a la anticoagulación con heparina en pacientes 
con hemorragia intraparenquimatosa. Es más segura en relación con la desestabilización medular en caso de lesiones medulares asociadas. Se evitan aumentos de la presión intracraneal por la posición del paciente y no es necesaria la intubación selectiva. Al no clamparse la aorta se elimina el riesgo de lesión isquémica de la médula espinal y del aumento de presión intracraneal.

Algunos autores realizan la colocación de la endoprótesis bajo anticoagulación con heparina ${ }^{4,14}$. A nuestros pacientes no se les realizó dicho tratamiento por riesgo de sangrado.

Las lesiones traumáticas de la aorta engloban desde el desgarro de la íntima, la rotura de aorta contenida o el seudoaneurisma hasta la sección completa de la aorta con sangrado activo. Malhotra et al definen la lesión aórtica mínima (LAM) como el desgarro de la íntima de menos de un centímetro, sin anomalías aórticas ni sangrado periaórtico ${ }^{7}$. Algunos autores defienden el tratamiento conservador de la LAM en pacientes hemodinámicamente estables realizando un seguimiento por TACHT o ECOT ${ }^{7,8}$.

Los 4 pacientes intervenidos tenían un seudoaneurisma de aorta con hematoma periaórtico asociado. En el caso no intervenido, la lesión corresponde a una LAM y fue disminuyendo hasta desaparecer en la angio-RMN a los 15 días. La resolución espontánea de esta lesión ya ha sido considerada en otros trabajos ${ }^{7,8}$.

No está aclarado el momento de realizar la intervención ${ }^{5,14}$. Una opción terapéutica es la reparación de forma diferida, priorizando las lesiones asociadas en caso de que supongan una amenaza vital para el paciente ${ }^{16,17}$. La intervención se podría realizar hasta 7 meses después del traumatismo ${ }^{5}$. Otros autores apoyan el tratamiento inmediato endovascular para un manejo más seguro ${ }^{14}$. Los pacientes intervenidos dentro de las primeras 36 horas y el intervenido a los 28 días evolucionaron bien posteriormente. En el operado tardíamente se hizo un seguimiento por ECOT.

En nuestros enfermos se realizó una oclusión intencionada de la arteria subclavia izquierda sin repercusión funcional. Otros autores ya publicaron esta misma técnica con el objetivo de una mejor fijación proximal de la prótesis y de evitar fugas periprotésicas. En caso de oclusión, el flujo de la vertebral y de la extremidad superior izquierda dependerá de arterias colaterales. La mayoría de los pacientes quedan asintomáticos y algunos refieren parestesia en la extremidad superior ${ }^{18,19}$.

Por nuestros resultados consideramos que en traumatismos graves las lesiones de aorta se deben descartar con una TACHT al ingreso. La colocación de una endoprótesis aórtica se debería hacer lo antes posible en caso de seudoaneurisma aórtico, siempre que se hayan estabilizado las lesiones asociadas que comprometan la vida. Si la lesión de aorta fuera mínima o se difiere la intervención por otra causa se ha de seguir la evolución por TACHT o ECOT. Se necesitarían estudios con un mayor número de casos para poder establecer cuál debería ser la actitud terapéutica en esta entidad.

\section{Declaración de conflicto de intereses}

Los autores han declarado no tener ningún conflicto de interés.

\section{BIBLIOGRAFÍA}

1. Parmley LF, Mattingly TW, Manion WC, Jahnke EJ. Nonpenetrating traumatic injury of the aorta. Circulation. 1958;17: 1086-101.

2. Smith MD, Cassidy JM, Souther S, Morris EJ, Sapin PM, Johnson SB, et al. Transesophageal echocardiography in the diagnosis of traumatic rupture of the aorta. N Engl J Med. 1995;332: 356-62.

3. Chip Jin NG, Jih Chang C, Li Jen W, Te Fa C, Pao Hsien C, Wen Huei L, et al. Diagnostic value of the helical CT for traumatic aortic injury: correlation with mortality and early rupture. J Emerg Med. 2006;30:277-82.

4. Broux C, Thony F, Chavanon O, Bach V, Hacicni R, Sengel $\mathrm{C}$, et al. Emergency endovascular stent graft for acute blunt thoracic aortic injury: a retrospective case control study. Intensive Care Med. 2006;32:770-4.

5. Reed AB, Thompson JK, Crafton CJ, Delvecchio C, Giglia JS. Timing of endovascular repair of blunt traumatic thoracic aortic transections. J Vasc Surg. 2006;43:684-8.

6. Hirose H, Gill IS, Malangoni MA. Nonoperative management of traumatic aortic injury. J Trauma. 2006;60:597-601.

7. Malhotra AK, Fabian TC, Croce MA, Weiman DS, Gavant ML, Pate JW. Minimal aortic injury: a lesion associated with advancing diagnostic techniques. J Trauma. 2001;51:1042-8.

8. Vignon P, Martaillé JF, François B, Rambaud G, Gastinne H. Transesophageal echocardiography and therapeutic management of blunt aortic injuries. J Trauma. 2005;58:1150-8.

9. Fishman J, Nunez D, Kane A, Rivas LA, Jacobs WE. Direct versus indirect signs of traumatic aortic injury reveled by helical CT: performance characteristics and interobserver agreement. AJR Am J Roentgenol. 1999;172:1027-3.

10. Fabian TC, Davis KA, Gavant ML, Croce MA, Melton SM, Patton JH Jr, et al. Prospective study of blunt aortic injury: helical $\mathrm{CT}$ is diagnostic and antihipertensive therapy reduces rupture. Ann Surg. 1998;227:666-76.

11. Pate JW, Fabian TC, Walker W. Traumatic rupture of the aortic isthmus: an emergency? World J Surg. 1995;19:119-26.

12. Akins CW, Buckley MJ, Dagget W, McIlduff JB, Austen WD. Acute traumatic disruption of the aorta: a ten- year experience. Ann Thorac Surg. 1981;31:305-9.

13. Dake MD, Miller DC, Semba CP, Mitchell RS, Walker PJ, Liddell RP. Transluminal placement of endovascular stent-grafts for treatment of descending thoracic aortic aneurysms. N Engl J Med. 1994;331:1729-34.

14. Agostinelli A, Saccani S, Borrello B, Nicolini F, Larini P, Gherli T. Inmediate endovascular treatment of blunt aortic injury: our therapeutic strategy. J Thorac Cardiovasc Surg. 2006;131: 1053-7.

15. Hutschala D, Fleck T, Czerny M, Ehrlich M, Schoder M, Lammer J, et al. Endoluminal stent- graft placement with acute aortic dissection type B. Eur J Cardiothorac Surg. 2002;21:964-9.

16. Kwon CC, Gill IS, Fallon WF, Yowler C, Akhrass R, Temes RT, et al. Delayed operative intervention in the management of traumatic descending thoracic aortic rupture. Ann Thorac Surg. 2002;74:1888-91.

17. Fattori R, Nienaber CA, Rousseau H, Beregi JP, Heijmen $\mathrm{R}$, Grabenwoger M, et al. Talent Thoracic Retrospective Registry. Results of endovascular repair of the thoracic aorta with the Talent Thoracic stent graft: the Talent Thoracic Retrospective Registry. J Thorac Cardiovasc Surg. 2006;132:332-9.

18 Lawlor DK, Ott M, Forbes TL, Kribs S, Harris KA, De Rose $\mathrm{G}$. Endovascular management of traumatic thoracic aortic injuries. Can J Surg. 2005;48:293-7.

19 Gorich J, Asquan Y, Seifarth H, Kramer S, Kapfer X, Orend $\mathrm{KH}$, et al. Initial experience with intentional stent-graft coverage of the subclavian artery during endovascular thoracic aortic repairs. J Endovasc Ther. 2002;9 Suppl 2:II39-43. 